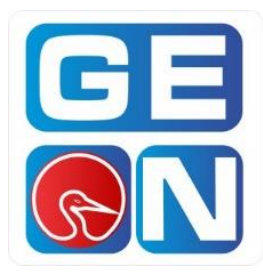

Revista GEON (Gestión, Organizaciones y Negocios.) ISSN: 2346-3910 en línea

revistageon@unillanos.edu.co

Universidad de los Llanos

Colombia

Gazca Herrera, Luis Alejandroi; Sánchez Hernández, Guillermo

Leonel ${ }^{\mathrm{ii}}$; Culebro Castillo, Karina; Zabala Arriola, Omariii.

Diagnóstico del uso de herramientas tecnológicas para la gestión y apoyo del liderazgo en las PYMES

Revista GEON, Vol. 6, No. 1, 2019

Pág. 69-83

Disponible en: $\underline{\text { https://doi.org/10.22579/23463910.85 }}$

\footnotetext{
' https://orcid.org/0000-0001-7637-2909

ii https://orcid.org/0000-0002-0607-9309

iii https://orcid.org/0000-0002-5754-547X
}

Esta publicación se encuentra bajo licencia: Creative Commons ReconocimientoNoComercialSinObraDerivada 4.0 Internacional
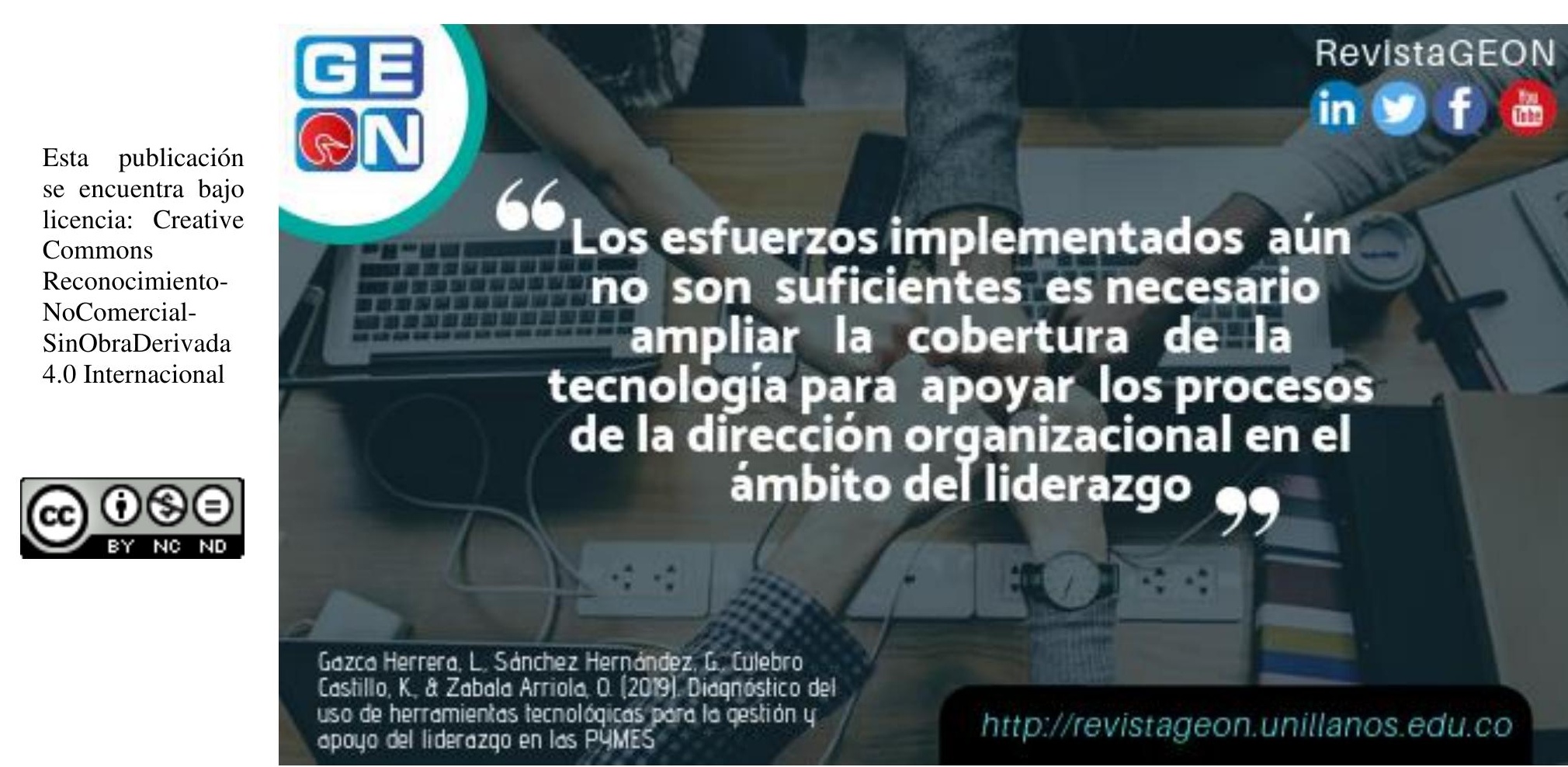


\title{
Diagnóstico del uso de herramientas tecnológicas para la gestión y apoyo del liderazgo en la PyMES
}

\section{Diagnosis of the use of technological tools for the management and support of leadership in the PyMES}

\author{
Luis Alejandro Gazca Herrera \\ lgazca@uv.mx
}

Licenciado en Sistemas Computacionales Administrativos, Maestro en Ciencias Administrativas y Doctor en Administración Pública, Universidad Veracruzana, México, @Alex_Gazca

\section{Guillermo Leonel Sánchez Hernández gusanchez@uv.mx}

Licenciado en Instrumentación Electrónica, Maestro en Tecnología Educativa y Doctor Planeación Estratégica y Dirección de Tecnologías, Universidad Veracruzana, México, @jalilsarj

\section{Karina Culebro Castillo}

kculebro@uv.mx

Licenciada en Administración, Maestra en Ciencias Administrativas y Doctora Administración y Desarrollo Empresarial, Universidad Veracruzana, México

\section{Omar Zabala Arriola}

ozabala@uv.mx

Licenciado en Estadística, Maestro en Negocios Internacionales, Universidad Veracruzana, México

Revista GEON Vol. 6 No 1 enero - junio 2019

ISSN 2346-3910 en línea http://revistageon.unillanos.edu.co/index.php/geon/article/view/85 


\section{Resumen}

Este artículo tiene como objetivo mostrar el resultado del diagnóstico sobre el uso de las Herramientas Tecnológicas (HT) para la gestión y apoyo del liderazgo en pequeñas y medianas empresas (PyMES) de la región de Xalapa, Veracruz México, pretendiendo señalar la pertinencia de esta tecnología ya que en años recientes se ha venido demostrando que las organizaciones de todo tipo y tamaño requieren de un cambio significativo en el enfoque de su propia gestión para lo cual es necesario la incorporación de las Tecnologías de la Información; el estudio se realizó con base en una muestra de empresas aplicando un instrumento que recopiló datos sobre los líderes y seguidores, evaluando cuántos de ellos utilizan herramientas tecnológicas y qué es lo que aportan en la mejora de sus actividades de liderazgo, con el fin de persuadir a sus colaboradores propiciando el trabajo en equipo para el logro de los objetivos de la empresa. Respaldando su importancia en el entorno organizacional, se analizan las teorías del liderazgo identificando sus características, sus actitudes y acciones pertinentes para mejorar su desempeño. Sin embargo, el uso de las HT ha sido poco utilizadas de tal forma que el conocimiento sobre el liderazgo no es suficiente para ejercerlo, es necesario el uso de la tecnología para mejorarlo.

Palabras claves: Liderazgo, Herramientas Tecnológicas, PyMES, Gestión

Abstract
The purpose of this article is to show the result of the diagnosis of the
use of the Technological Tools (TH) for the management and support of
small and medium-sized enterprises (SMEs) in the Xalapa region,
Veracruz México, aiming to point out the relevance of this technology
since in recent years it has been demonstrated that organizations of all
types and sizes require a significant change in the focus of their own
management, which requires the incorporation of Information
Technologies, the study was conducted based on A sample of companies
applying an instrument that collected data on leaders and followers,
evaluating how many of them use technological tools and what they
contribute to improving their leadership activities, in order to persuade
their collaborators by promoting work in equipment to achieve the
objectives of the company. Understanding its importance in the
organizational environment, the theories of leadership are analyzed
identifying their characteristics, attitudes and actions relevant to


improving their performance. However, the use of the TH has been little used in such a way that knowledge about leadership is not enough to exercise it, it is necessary to use the technology to improve it.

Keywords: Leadership, Technological Tools, SMEs, Management

\section{Code JEL: M1 Business Administration}

\section{Introducción}

El sector económico de México, a pesar de estar fuertemente movido por empresas transnacionales, sigue siendo conformado en su mayoría por negocios de escala mucho menor, administrados por grupos reducidos y con menores recursos, y que, a pesar de esto, aportan de manera significativa al desarrollo del país. De acuerdo al Instituto Nacional de Estadística y Geografía (INEGI), al otrora Instituto Nacional del Emprendedor (INADEM) y el Banco Nacional de Comercio Exterior (BANCOMEXT), realizaron la Encuesta Nacional sobre Productividad y Competitividad de las Micro, Pequeñas y Medianas Empresas ENAPROCE 2015, cuyo resultado demostró que, del total de empresas consideradas, $97.6 \%$ son microempresas y concentran el $75.4 \%$ del personal ocupado total. Le siguen las empresas pequeñas, que son un $2 \%$ y tienen el $13.5 \%$ del personal ocupado. Las medianas representan $0.4 \%$ de las unidades económicas y tienen poco más del $11 \%$ de los ocupados (Bancomext 2015).

De las microempresas el $74.5 \%$ no cuentan con equipo de cómputo por razones como: falta de recursos, no la necesitan, no saben usarlos, entre otros, en el mismo sentido el $73.9 \%$ no tienen conectividad a internet por situaciones similares a las anteriores. Y de acuerdo a una investigación de Forbes nos dice que solo el 16\% de las PyMES invierte en tecnología y talento (Forbes 2018).

\begin{tabular}{|l|r|r|r|}
\hline \multirow{2}{*}{ Tamaño } & \multicolumn{2}{|c|}{ Empresas } & \multicolumn{1}{c|}{$\begin{array}{c}\text { Personal } \\
\text { ocupado }\end{array}$} \\
\cline { 2 - 4 } & Número & $\begin{array}{c}\text { Participación } \\
(\%)\end{array}$ & $\begin{array}{c}\text { Participación } \\
\text { (\%) }\end{array}$ \\
\hline Micro & $3,952,422$ & 97.6 & 75.4 \\
\hline Pequeña & 79,367 & 2.0 & 13.5 \\
\hline Mediana & 16,754 & 0.4 & 11.1 \\
\hline Total & $\mathbf{4 , 0 4 8 , 5 4 3}$ & $\mathbf{1 0 0 . 0}$ & $\mathbf{1 0 0 . 0}$ \\
\hline
\end{tabular}

Tabla 1.- Número de empresas por tamaño y personal ocupado

Fuente: Bancomext (2015)

Como complemento a lo antes mencionado a partir del segundo semestre del 2009 la Secretaría de Hacienda y Crédito Público (SHCP) y la Secretaría de Economía (SE) establecieron las bases para una nueva clasificación de acuerdo a sus características en cuanto al número de empleados y a las ventas que realizan anualmente.

Es así como (Paredes 2018) define esa clasificación que a continuación se describe; las microempresas en México son las que se encuentran en todos los 
sectores de negocio y cuenta con hasta diez trabajadores. Su monto de ventas anuales es de un aproximado de 4 millones de pesos.

Las pequeñas empresas son aquellas que se dedican al sector del comercio, la industria y servicios; cuentan de 11 hasta 30 trabajadores y sus ventas anuales son de más de cuatro y hasta cien millones de pesos anuales. Las medianas empresas se dividen en tres sectores, en el comercio, servicios e industria, en el primer caso el número de trabajadores es de 31 hasta 100 ; sus ventas anuales son desde más de 100 millones y menos de 250 millones de pesos; en el segundo y tercer sector, el número de trabajadores es de 51 hasta 250 y en ambos casos; el monto de ventas anuales tiene un rango de 100.1 hasta 250 millones de pesos (Paredes 2018).

Considerando dichos porcentajes, se puede identificar la importancia de tener conocimiento sobre las tendencias de desarrollo que las MiPymes presentan, con el fin de monitorearlas y buscar formas en las cuales se pueda estimular su crecimiento, fortaleciendo así la economía del país. Para su propio desarrollo, todas las empresas requieren del aprovechamiento de sus recursos, encontrándose, entre ellos el capital humano, cuya adecuada dirección es imprescindible para alcanzar los objetivos de la empresa.

De esta manera, el liderazgo se convierte en un punto medular del ámbito empresarial, y una forma de apoyarlo es aprovechando herramientas tecnológicas que apoyen y faciliten la gestión del líder.

\section{Contexto teórico}

El liderazgo ha sido parte medular de la historia humana y objeto de análisis desde hace cientos de años, más su estudio formal no comenzó hasta inicios del siglo $\mathrm{XX}$, cuando surgieron las primeras teorías que buscaron definir el liderazgo y las distintas formas en las cuales se manifiesta. Tales teorías evolucionaron a lo largo del siglo, modificándose $\mathrm{y}$ complementándose entre ellas, y en dicha evolución se pueden identificar cuatro tendencias o enfoques principales, de rasgos, conductual, situacional y transformacional. El estudio de esta investigación consideró tres teorías de liderazgo que a continuación se mencionan. Teoría del liderazgo Laissezfaire, Guirriman (2016) brinda un concepto básico sobre esta teoría, se basa en el supuesto de ser un tipo de liderazgo pasivo/evitador, es un liderazgo donde los propios colaboradores establecen sus objetivos con el simple hecho de lograr la aplicación del plan estratégico, por tal puede tomarse como aquel líder que evade la toma directa de decisiones y no hace uso de su autoridad. Teoría del liderazgo transaccional, en este estilo el líder hace uso de su posición de poder para mantener el flujo normal de las operaciones, recompensando o castigando a sus subordinados con base en su rendimiento previamente acordado Almiron, Tikhomirova y García, 2015). Teoría del liderazgo transformacional, (Bresó, 2013) nos muestra esta teoría como un énfasis para que los líderes aprendan a ejercer la influencia en la motivación de sus colaboradores, se expresa como un esfuerzo extra por parte de sus 
colaboradores en el ejercicio de inspiración y consideración individualizada.

Ahora bien, en cuanto al aspecto de las TI, (Guisao et. al. 2017) citando a (WEF 2015) menciona que en el entorno globalizado las organizaciones deben fortalecer las estrategias de implementación y desarrollo de las TI con el fin de optimizar y robustecer cada uno de los procesos y generar valor, que se traduce en rendimiento y competitividad. Por ende, reitera (Guisao et. al. 2017), que en la literatura ha surgido un interés por explorar el impacto de la relación entre las capacidades de tecnologías de información (CTI) y el desempeño organizacional (DO) el cual mucho tiene que ver con la cuestión de liderazgo y la forma como el líder influye sobre sus colaboradores (Bharadwaj, 2000; Santhanam y Hartono, 2003; Liu et al., 2013), entendidas las primeras como la capacidad de una empresa para adquirir, combinar, reconfigurar, movilizar y desplegar constantemente los recursos de TI en respuesta al entorno dinámico para mejorar los procesos de trabajo $y$ estrategias de negocio, y así generar valor agregado y lograr una ventaja competitiva difícil de imitar o sustituir. A su vez, el DO se entiende como un constructo multidimensional compuesto por un conjunto de resultados objetivos y subjetivos que son medidos por indicadores financieros y no financieros del negocio (Nakata et al., 2008; Liu et al., 2013).

(Guisao et. al. 2017) en la investigación que llevó a cabo menciona que los estudios sobre la relación entre
CTI y DO han logrado demostrar que esta habilidad organizacional puede proporcionar mayores beneficios económicos, reducción de costos de comercialización (Chae et al., 2014), y por ende un rendimiento financiero superior (Bharadwaj, 2000). Continúa estableciendo que, por otro lado, estudios recientes presentan resultados contradictorios que indican la inexistencia de la relación entre CTI y DO, dado que, a medida que aumenta la accesibilidad y disponibilidad de las TI y su costo disminuye, se convierten en un estándar de la industria, sin posibilidades de proporcionar diferenciación (Chae et al., 2014). Sin embargo, estos estudios se han desarrollado en países con un alto grado de incorporación de TIC en los negocios en comparación con países emergentes como lo es México, dado que en el caso de los primeros en años recientes el índice de disponibilidad de TI ha estado por encima de 5,0 mientras que en los segundos se ha mantenido entre 3,5 y 3,8 (WEF, 2015). Por esto, sería pertinente contar con estudios que aporten evidencia empírica concluyente en torno a esta relación en el contexto de países emergentes, en donde las TI sí podrían seguir siendo una fuente de ventaja competitiva (Liu et al., 2008).

\section{Marco Referencial}

La ciudad de Xalapa, capital del estado de Veracruz. Está ubicada al centro del estado y aproximadamente 350 kilómetros al este de la Ciudad de México, colinda al norte con Banderilla, Jilotepec y Naolinco, al sur con Coatepec, al oeste con Tlalnelhuayocan y al este con Actopan y Emiliano Zapata. Sus $124.38 \mathrm{~km}^{2}$ de extensión representan el $0.17 \%$ del 
territorio veracruzano. En la ciudad de Xalapa, existen aproximadamente 26,463 micro, pequeñas y medianas empresas registradas (MiPymes:2017), de las cuales, Según datos recabados por el Directorio Estadístico Nacional de Unidades Económicas, los ramos de las empresas con mayor número de registros se dividen en cinco categorías: a) Comercio minorista y mayorista; b) Transportes, correos y almacenamiento; c) Servicios inmobiliarios y de alquiler de bienes inmuebles e intangibles; d) Servicios de esparcimiento y recreativos; $y$ e) Servicios de alojamiento temporal y de preparación de alimentos y bebidas.

\section{Materiales y métodos}

Debido a la naturaleza estadística, ordenada y secuencia de la investigación, el enfoque utilizado es de tipo cuantitativo. Desde el inicio, a partir de una idea y posterior identificación de un problema, se logró establecer una serie de "objetivos, preguntas, justificación, viabilidad y evaluación de las deficiencias", elementos que, de acuerdo con (Sampieri 2006), son necesarios para considerar una investigación de dicho tipo. De igual forma para la realización de la presente investigación, se utilizó el método de investigación documental, empleando la técnica de sistematización.

Con base en los datos de la ciudad de Xalapa donde se cuentan con 26,463 MiPymes y con base en los porcentajes del ENAPROCE, podemos aproximar que 25,828 son microempresas, 529 son pequeñas empresas y 106 son empresas medianas. De estos datos a continuación se mencionan porcentajes de acciones que realizan las empresas en cuanto al ámbito de la dirección organizacional considerando aspectos como el de capacitación, liderazgo o supervisión de acuerdo con ENAPROCE, de tal forma que el $73.7 \%$ de las empresas medianas realiza acciones de dirección es decir 78 empresas, de las pequeñas empresas el $55.8 \%$ traducido como 295 empresas y las microempresas 2,970 empresas las llevan a cabo (Datos calculados propiamente por método estadístico de porcentajes aplicados).

Las capacidades de una empresa ante cualquier situación son de suma importancia en cuanto a liderazgo y dirección se trata, la misma encuesta nos menciona que de un conjunto de empresas encuestadas, el $16.6 \%$ pueden dar solución a las situaciones sin llevar acciones posteriores, el $26.20 \%$ da solución llevando a cabo medidas posteriores para evitarlos, el $10.34 \%$ dan solución e instrumentación y mejora continua y un $46.83 \%$ no da solución a las situaciones. Ahora bien, dada las características de las MiPymes la presente investigación se enfocó sobre las pequeñas y medianas empresas que son las que básicamente pueden implementar tecnología, por tal motivo de las 373 empresas potenciales a ser nuestra población nos interesó considerar aquellas que toman acciones y se preocupan en la mejora continua como parte de su desarrollo organizacional por tal motivo se consideró el $36.54 \%$ sobre las que se realizará la aplicación de los instrumentos. Como resultado tenemos 97 empresas que toman decisiones con base en situaciones presentadas y 39 empresas que tienen mejora continua. Esto significa 
que contamos con 136 empresas de población potencial. Consideramos de igual importancia el ambiente de trabajo y su regulación como empresa, en donde la misma empresa decide que hacer.

De las 136 empresas el $83.2 \%$ es decir 113 empresas de nuestra población potencial se dedican a seguir progresando como negocio y seguir creciendo. Al ser esta una investigación de herramientas tecnológicas tomamos de la encuesta dictada anteriormente, el $27.1 \%$ del total de empresas ocupan equipo y herramientas de cómputo por tal motivo de las 113 empresas de población potencial existe la posibilidad de que 31 empresas de la ciudad de Xalapa cuentan con uso de estas tecnologías. Al estar en una era de comunicación basada en tecnologías y cercanos al internet de las cosas, por obvias razones las nuevas herramientas que se pueden apoyar en la gestión y evaluación de liderazgo en dichas empresas necesitan $\mathrm{o}$ puede ser dependiente del internet. En la misma encuesta, nos indica que del total de empresas el $27.6 \%$ hacen uso de internet, para sacar nuestra población potencial final, de las 31 empresas se estima que 9 de estas entre pequeñas y medianas de Xalapa son las ideales para poder generar una investigación directa y precisa, por lo que se consideran 8 pequeñas empresas $y$ 1 empresa mediana para poder evaluar la investigación. Dado que la investigación se enfoca en la identificación de la aplicación de las tecnologías de la información que apoyan en la gestión y liderazgo es competente ver los dos puntos o las dos previas partes de una organización, los jefes o personas de rango superior y carácter organizacional o directivo y a los colaboradores o demás miembros de la estructura organizacional de la empresa, viendo y observando cuáles son sus puntos de vista sobre las cuestiones que se aplicarán en el instrumento de recolección. Así mismo la investigación consideró de mayor atención a empresas de negocios comerciales donde el contacto entre los directivos, jefes o gerentes es más cercano a sus colaboradores, miembros o subordinados de trabajo.

Ahora bien, considerando a 9 empresas donde se llevó a cabo la investigación, dada esta situación el cálculo de la muestra se tomó en cuenta al número de empleados a los cuales se aplicará el instrumento de recolección de datos.

Dado que de esas empresas no se conocía el número de empleados se aplicó la fórmula para una población infinita. Anderson, Sweeney y .Williams. (2008), Nos dicen "El tamaño de la muestra para una estimación por intervalo de la proporción poblacional, se determina con una fórmula para población infinita" ( $p$. 321)

$$
n=\frac{\left(Z_{\alpha / 2}\right)^{2} P(1-P)}{E^{2}}
$$

Dónde:

$Z_{\alpha / 2}$ Es el nivel de confianza (1.96)

$P=\quad$ Es el nivel de éxito

$(1-P)=\quad$ Es la probabilidad total menos el éxito, es decir, el fracaso

$E^{2}=\quad$ Es el error muestral

$$
n=\frac{(1.96)^{2}(.5)(.5)}{(0.10)^{2}}=96
$$


96 sería el número de participantes para los cuales habría que aplicar encuestas a las 9 empresas, 8 pequeñas y 1 mediana, quedarían distribuidas de la siguiente manera:

$$
\left(\frac{1}{9}\right) 96=11 \quad\left(\frac{8}{9}\right) 96=85
$$

Con base en los cálculos se debe aplicar el instrumento a 96 participantes, de los cuales 11 deben ser subordinados y jefes de una empresa mediana y 85 de empresas pequeñas.

Otro aspecto importante de la investigación fue el diseño del instrumento que se aplicó a los líderes y a los seguidores para determinar cómo apoyan las Tecnologías de información a la gestión del liderazgo, para ello se tuvo que realizar una precisa delimitación del objeto de interés dando con ello el comienzo de la conceptualización para determinar de manera concreta qué es lo que se iba a medir (Becker 1998). Para llegar a la conceptualización se llegó de la teoría a través de un proceso deductivo en el que se extrajo el constructo y los indicadores a medir. Finalmente se realizó el proceso de la conceptualización al de operacionalización, el cual fue a través del paso de la variable teórica (uso de la TI en la gestión y apoyo al liderazgo) a indicadores empíricos verificables y medibles en ítems. Se diseñaron dos instrumentos uno para los líderes con una variable, tres dimensiones, al menos dos indicadores por dimensión y por lo menos tres ítems por cada indicador; para los seguidores (subordinados) el instrumento estaba compuesto por una variable, dos dimensiones, al menos 3 indicadores por dimensión y por lo menos cuatro ítems por indicador.

Posterior la operacionalización de los instrumentos se llevó a cabo la propuesta de codificación para cada una de las categorías y finalmente el nivel de medición que fue por escala de Likert. Una vez diseñado el instrumento fue validado por juicio de expertos el cual es un método de validación muy útil para verificar la fiabilidad de una investigación el cual según (Cabero y Llorente, 2013) citado por (Garrote y Rojas, 2015) consiste, básicamente, en solicitar a una serie de personas para este caso a cinco expertos, la demanda de un juicio hacia el instrumento, se consideraron cuatro categorías para el juicio de expertos las cuales fueron suficiencia, coherencia, relevancia y claridad; el resultado fue favorable haciendo modificaciones mínimas sobre todo en la categoría de coherencia (Escobar 2008).

Posterior a la validez del instrumento se aplicó un pilotaje para aplicarle Coeficiente alfa de Cronbach para medir la confiabilidad del tipo de consistencia interna de la escala establecida, es decir, para evaluar la magnitud en que los ítems del instrumento están correlacionados. Las tablas 3 y 5 representa la fiabilidad del instrumento de obtención de información el cual tiene un valor de alfa de Cronbach de .729 y .712 lo que representa un valor aceptable del instrumento según George y Mallery (2003, p. 231).

- Coeficiente alfa $>.9$ es excelente

- Coeficiente alfa $>.8$ es bueno

- Coeficiente alfa >.7 es aceptable

- Coeficiente alfa >.6 es cuestionable

- Coeficiente alfa $>.5$ es pobre 
- Coeficiente alfa <.5 es inaceptable

Análisis de Confiabilidad

a. Eliminación por lista basada en todas las variables del procedimiento.

\begin{tabular}{|ll|r|l|}
\hline & $\mathrm{N}$ & \multicolumn{1}{|c|}{$\%$} \\
\hline \multirow{4}{*}{ Casos } & Válidos & 17 & 100.0 \\
& Excluidos & 0 & .0 \\
& Total & 17 & 100.0 \\
\hline
\end{tabular}

Tabla 2. Resumen del procesamiento de los casos de las variables para los líderes.

Fuente: Elaboración propia SPSS V. 21 (2018)

\section{Estadísticos de fiabilidad}

\begin{tabular}{|r|r|}
\hline Alfa de Cronbach & No. de elementos \\
\hline .729 & 19 \\
\hline
\end{tabular}

Tabla 3. Alfa para instrumento líder.

Fuente: Elaboración propia SPSS V. 21

(2018)

Análisis de Confiabilidad

a. Eliminación por lista basada en todas las variables del procedimiento.

\begin{tabular}{|rl|r|l|}
\hline & $\mathrm{N}$ & \multicolumn{1}{|c|}{$\%$} \\
\hline \multirow{4}{*}{ Casos } & Válidos & 79 & 100.0 \\
& Excluidos & 0 & .0 \\
& Total & 79 & 100.0 \\
\hline
\end{tabular}

Tabla 4. Resumen del procesamiento de los casos de las variables para los seguidores.

Fuente: Elaboración propia SPSS V. 21 (2018)

\section{Estadísticos de fiabilidad}

\begin{tabular}{|r|r|}
\hline Alfa de Cronbach & No. de elementos \\
\hline .712 & \\
\hline
\end{tabular}

Tabla 5. Alfa para instrumento seguidor.

Fuente: Elaboración propia SPSS V. 21 (2018)

La aplicación del instrumento se llevó acabo de manera simultánea con las empresas; en la mayoría de ellas se procuró cubrir un promedio de 1 a 3 empleados con cargo de liderazgo, es decir algún cargo que estuviese al mando de personal; en cuanto a demás empleados (seguidores o subordinados) se procuró cubrir un promedio de 8 a 10 empleados.

\section{Resultados}

A continuación, se muestran los resultados más significativos con respecto a los líderes con respecto a las herramientas tecnológicas para el apoyo de sus acciones.

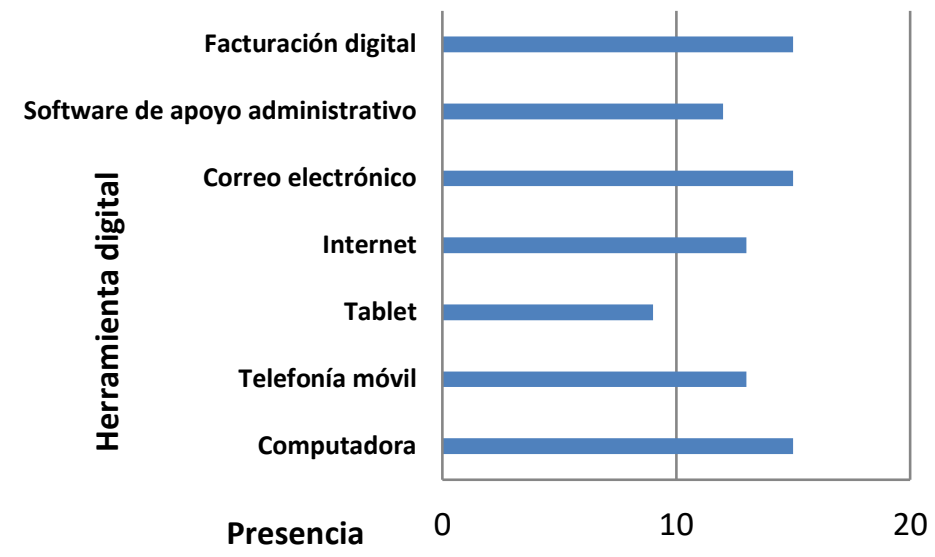

Gráfica 1.- Presencias de herramientas tecnológicas en las PyMES

Fuente: Encuestas aplicadas elaboración propia (2018)

En la gráfica 1, se puede apreciar que las PyMES hacen uso de más de un equipo o herramienta tecnológica para apoyar los procesos administrativos, que va desde el uso de computadora hasta el software de apoyo administrativo por lo que se visualiza el uso tecnológico en las PyMES

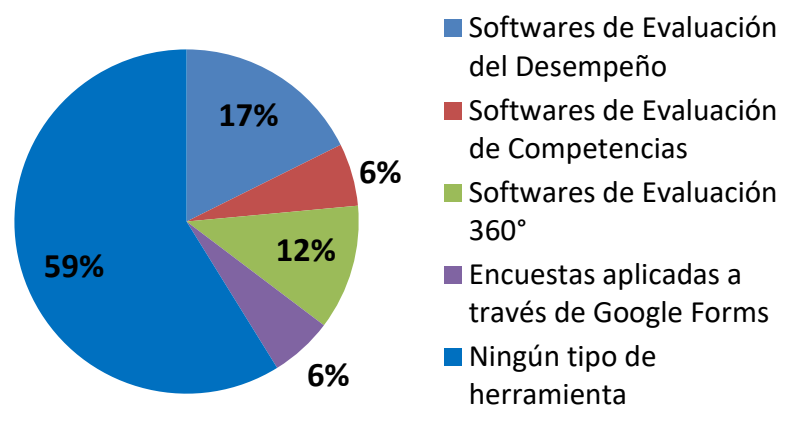

Gráfica 2.- Mecanismos o herramientas tecnológicas utilizadas para el apoyo o gestión del liderazgo en sus trabajadores

Fuente: Encuestas aplicadas elaboración propia (2018) 
En la gráfica 2, se puede visualizar que el $59 \%$ de los líderes encuestados no aplican ningún tipo de herramienta tecnológica que les apoye para la mejora o gestión del liderazgo sobre sus colaboradores, un $17 \%$ utiliza software de evaluación del desempeño, un $12 \%$ software de evaluación $360^{\circ}$ y el resto del porcentaje algún tipo de aplicación, sin embargo, se puede destacar que 6 de cada 10 líderes no hacen uso de la tecnología para apoyarle en sus actividades de dirección.

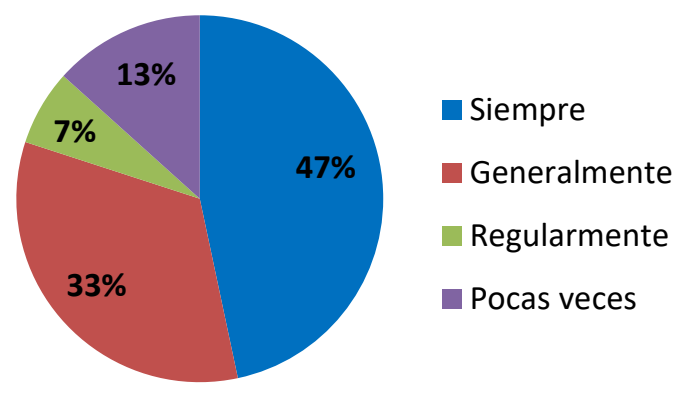

Gráfica 3.- Frecuencia de aplicación del uso herramientas tecnológicas utilizadas para el apoyo o gestión del liderazgo en sus trabajadores

Fuente: Encuestas aplicadas elaboración propia (2018)

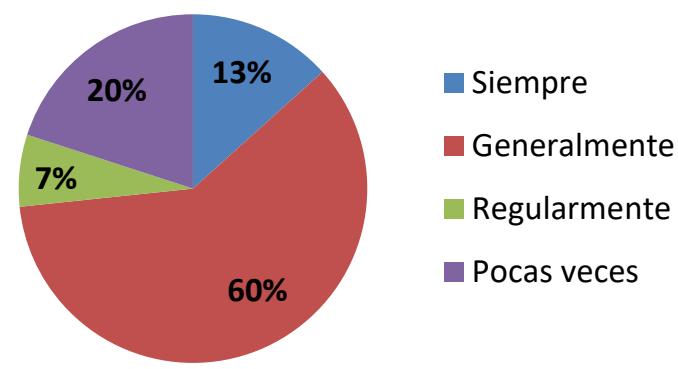

Gráfica 4.- Asistencia a capacitación de líderes para la implementación de herramientas tecnológicas.

Fuente: Encuestas aplicadas elaboración propia (2018)

Del casi el $40 \%$ de los líderes que utilizan herramientas tecnológicas para el apoyo o gestión del liderazgo y como se puede apreciar en la gráfica 4, el $47 \%$ las utiliza con una frecuencia de siempre, un $33 \%$ generalmente, un $13 \%$ pocas veces y un $7 \%$ pocas veces, lo que demuestra que seis de cada diez líderes si hacen un uso frecuente de dichas herramientas.

Como se puede visualizar en la gráfica 4 , el $73 \%$ de los líderes asisten a capacitación en temas que tiene que ver con la implementación de herramientas tecnológicas para mejorar los aspectos de liderazgo en la empresa, lo que significa que existe preocupación por una capacitación constante en dicho rubro.

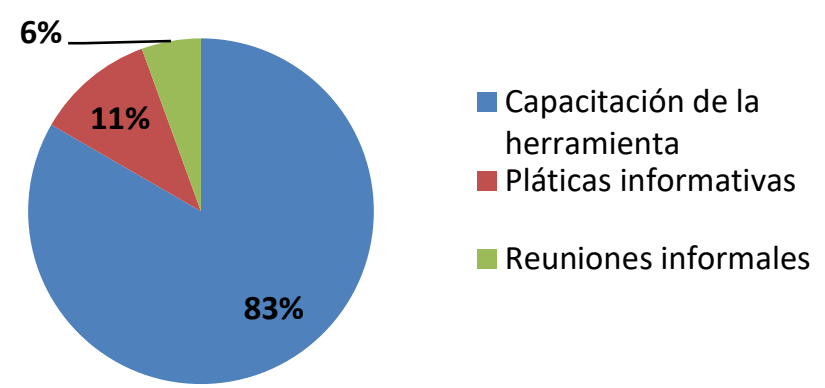

Gráfica 5.- Cómo se informa a los seguidores del uso de herramienta tecnológica que apoyan las actividades de liderazgo

Fuente: Encuestas aplicadas elaboración propia (2018)

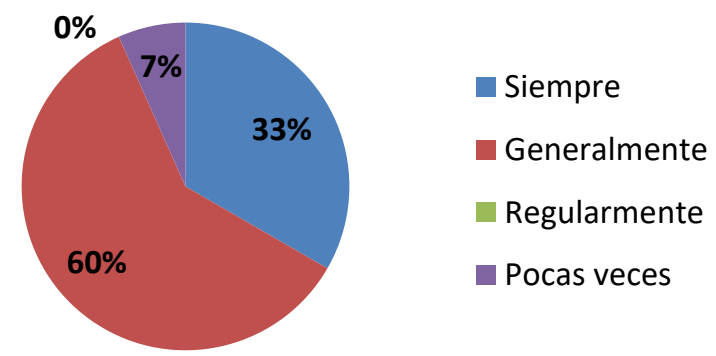

Gráfica 6.- Cómo consideran los directivos que el uso de las herramientas tecnológicas dirigidas al liderazgo contribuye al éxito de la empresa

Fuente: Encuestas aplicadas elaboración propia (2018)

Como se puede apreciar en la gráfica 5 el $83 \%$ de los líderes informan a los seguidores por medio de la capacitación de 
las herramientas tecnológicas que utilizan para los procesos de evaluación de estos, un $11 \%$ lo realiza con pláticas informativas y solo un $6 \%$ con pláticas informarles, se considera este un rubro importante ya que es necesario que los seguidores conozcan las herramientas que los líderes utilizan para evaluarlos.

La gráfica 6 muestra que el 93\% de los directivos considera que siempre o generalmente las herramientas tecnológicas dirigidas al liderazgo contribuyen al éxito de la empresa, sin embargo, este porcentaje tan alto debe ser considerado para la implementación de estas que como se mostró en la gráfica 3 , casi el $60 \%$ de las empresas no utilizan dichas herramientas.
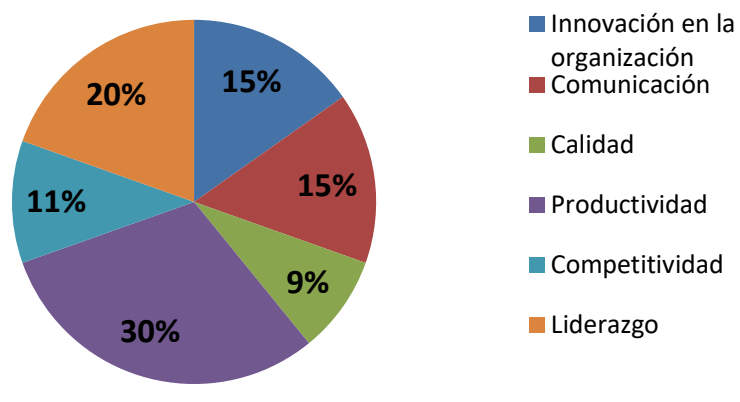

Gráfica 7.- Factores que los líderes consideran mejorarían con la implementación de HT.

Fuente: Encuestas aplicadas elaboración propia (2018)

Como se puede apreciar en la gráfica 7 un aspecto significativo es que el $20 \%$ de los encuestados considera que uno de los factores que mejorarían con la implementación de las herramientas tecnológicas es el liderazgo, por lo que su implementación debe ser considerada como una prioridad para mejorar dicha actividad.
A continuación, se muestran los resultados más significativos con respecto a los seguidores (subordinados) con respecto a las herramientas tecnológicas que aplican sus líderes.

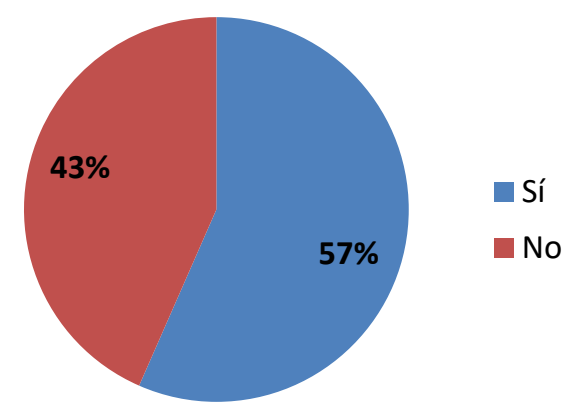

Gráfica 8.- Ha identificado mecanismos que mejoran los procesos de liderazgo que le permite retroalimentar a sus superiores.

Fuente: Encuestas aplicadas elaboración propia (2018)

Como se aprecia en la gráfica 8 , el $57 \%$ de los seguidores identifican mecanismos que mejoran los procesos de liderazgo.

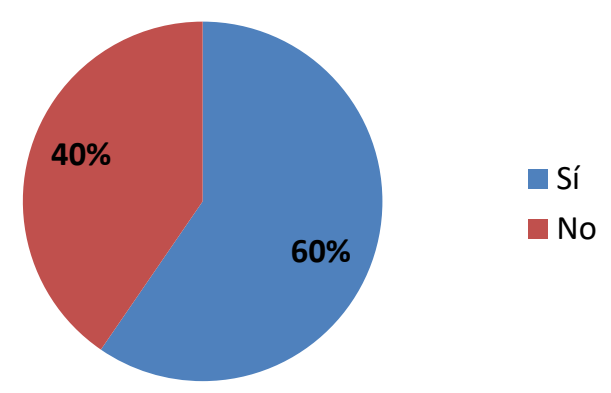

Gráfica 9.- ¿Los mecanismos implementados hacen uso de las herramientas tecnológicas?

Fuente: Encuestas aplicadas elaboración propia (2018)

Como se visualiza en la gráfica 9 , del $60 \%$ de seguidores que identifican mecanismos para mejorar los procesos de liderazgo. 


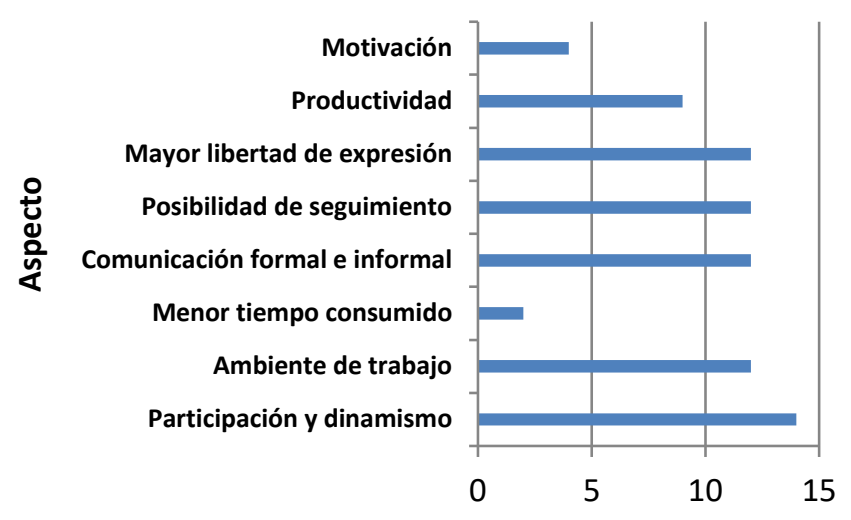

Gráfica 10.- Aspectos que mejoran con el uso de las herramientas tecnológicas en los procesos de liderazgo

Fuente: Encuestas aplicadas elaboración propia (2018)

Como se visualiza en la gráfica 10 , son diversos los aspectos que los seguidores consideran que mejoran con el uso de las herramientas tecnológicas, entre ellos destacar la participación, el dinamismo la comunicación y el seguimiento de sus actividades.

\section{Conclusiones}

En la mayoría de las empresas se pudo apreciar que por parte del directivo aplica los métodos para poder liderar, guiar y dirigir la empresa o el personal a su cargo, un porcentaje no significativo de estos líderes cuentan con las herramientas tecnológicas para mejorar sus aspectos de liderazgo.

Por parte de los seguidores se observó que un gran número de ellos identifican plenamente con qué tipo de líder están tratando, pero además se les informan sobre la forma en que emplean las herramientas tecnológicas para los procesos de comunicación, retroalimentación o evaluación, los cuales les son aplicados.
Con base en los resultados, las herramientas tecnológicas influyen en un porcentaje significativo en cómo pueden apoyar o mejorar las actividades de liderazgo, por tal motivo la mayor implementación de estas puede incrementar la gestión y el control de este, volviéndolo más intuitivo y apegado a la realidad, contando con información fidedigna que le apoye en la toma de decisiones en cuanto a la productividad de sus seguidores.

Sin embargo los esfuerzos implementados aún no son suficientes es necesario ampliar la cobertura de la tecnología para apoyar los procesos de la dirección organizacional en el ámbito del liderazgo, por tal motivo hacemos mención de algunas herramientas de software que les apoyen en la toma de decisiones sobre los recursos humanos, sistemas ampliados a evaluaciones psicométricas de los empleados, sistemas de control de rutinas y trabajo, sistemas de información con base en herramientas de rotación de personal basados en sus cualidades y experiencia de la empresa, entre otros.

Las propuestas de algunas herramientas tecnológicas basados en sistemas de información son los siguientes:

SimpleHRM, un sistema Open Source con el cuál se podrá gestionar de manera amplia y eficiente los procesos relacionados con los recursos humanos, tales como seguimiento de la información de los empleados, analizar y crear estadísticas de los empleados, lo que 
simplificará trámites y la toma de decisiones. (Díaz, 2011)

Orange HRM, es un sistema open source destinado a MIPYMES, el cual agiliza todo el proceso administrativo de la empresa, destinando cinco módulos para su gestión. Un módulo de reclutamiento; encargado de llevar un listado de las ofertas de trabajo y selección de personal, guiando el proceso de contratación. Un módulo de administración, parte en la que el administrador de recursos humanos trabajará las tareas de administración dentro del sistema. Un módulo de información de personal (PIM), mantiene la información de los empleados para uso de la administración. Un módulo de la licencia, Encargado de gestión de licencia, con amplias posibilidades de definir los tipos de licencia y más. Un módulo de prestaciones, en este módulo se ilustra la empresa del plan de salud (Diaz, Emprendices 2011). Este sistema de código libre simplificaría la mayoría de las tareas en la empresa, creando un vínculo más cercano entre el líder y sus colaboradores.

En el ámbito de la motivación también puede aplicar la tecnología, con software de calificación de empleados, con guías o manuales en línea o electrónicos que permitan al usuario hacer más entendible su trabajo y motivarse a realizarlo, un empleado que no sabe qué hacer al $100 \%$ en su trabajo es un empleado que no será productivo al 100\%.

Podemos concluir que las herramientas tecnológicas traerán beneficios en la forma en que los líderes realicen sus actividades, sin embargo la tecnología solo es un apoyo a la gestión, el verdadero tipo de liderazgo que se debe aplicar es el liderazgo transformacional, adaptable a todo tipo de situación, no casarse con un tipo de liderazgo, porque las situaciones en una empresa pueden cambiar repentinamente, y un buen líder debe saber sobrellevar la situación, sabiendo utilizar las herramientas con las que cuenta y el personal que lidera, para tener así en conjunto un buen ambiente de trabajo y productividad en la empresa.

\section{Referencias}

Almirón-Arévalo, V., Tikhomirova, A., \& García-Ramírez., A. T.-T. (2015). Liderazgo transaccional vs liderazgo transformacional. Reidocrea, 24-27.

Anderson, David R., Dennis J. Sweeney y Thomas A.Williams (2008). Estadística para administración y economía, México D.F. Cengage Learning Editores, S.A. de C.V.

Bada, L., Rivas, L. \& Littlewood, H. (2017). Modelo de asociatividad en la cadena productiva en las Mipymes agroindustriales. ELSEVIER, 62(4), 1059-1422. Obtenido de http://www.elsevier.es/es-revistacontaduria-administracion-87-resumenmodelo-asociatividad-cadena-productivalas-S0186104217300682\#bib0135

Bancomext (2015), recuperado el 12 de septiembre de 2018, de Bancomext: https://www.bancomext.com/comunicados/1 4237

Becker H. S. (1998): Tricks of the trade. How to think about your research while your re doing it. Chicago University Press. Chicago. 
Bharadwaj, A. S. (2000). A resource-based perspective on information technology capability and firm performance: An empirical investigation. MIS Quarterly, 24(1), 169-196. https://doi: $10.2307 / 3250983$

Bresó, R. G. (2013). ¿Es el liderazgo transformacional determinante en la motivación intrínseca de los seguidores? SCIELO, 59-64.

Cabero Almenara, J. y Llorente Cejudo, M. C. (2013), La aplicación del juicio de experto como técnica de evaluación de las tecnologías de la información (TIC). En Eduweb. Revista de Tecnología de Información y Comunicación en Educación, 7 (2) pp.11-22. Recuperado de:

http://tecnologiaedu.us.es/tecnoedu/imag es/stories/jca107.pdf

Díaz, J. (10 de 06 de 2011 A). Emprendices. Obtenido de Orange HRM: Programa gratuito para administrar los Recursos Humanos en una empresa: https://www.emprendices.co/orangehrm-programa-gratuito-para-administrarlos-recursos-humanos-en-una-empresa/

Díaz, J. (10 de 06 de 2011 B). Emprendices. Obtenido de Simple HRM: Software gratuito para la administración de Recursos Humanos: https://www.emprendices.co/simple-hrmsoftware-gratuito-para-la-administracionde-recursos-humanos/

Díaz, J. (23 de 01 de 2011 C). NominaSOL: Software gratuito de gestión de nóminas y seguros sociales para empresas. Obtenido de Emprendices: https://www.emprendices.co/nominasol- software-gratuito-de-gestion-de-nominasy-seguros-sociales-para-empresas/

Chae, H., Koh, C. \& Prybutok, V. (2014). Information technology capability and firm performance: Contradictory findings and their possible causes. MIS Quarterly, 38(1), 305-326. https://doi:10.25300/MISQ/2014/38.1.14

Escobar-Pérez, J.; Cuervo-Martínez, Á.: Validez De Contenido Y Juicio De Expertos: Una Aproximación a Su Utilización. Avances En Medición, 6, 27-36 (2008)

Forbes (2018), recuperado el 12 de julio de 2018, de Forbes: https://www.forbes.com.mx/pymesmexicanas-un-panorama-para-2018/

George, D. y Mallery, P. (2003). spss for Windows step by step: A Simple Guide and Reference. 11.0 Update (4. ${ }^{\mathrm{a}}$ ed.). Boston: Allyn \& Bacon.

Guirriman, a. a. (2016). Análisis Exploratorio de las Percepciones sobre los Estilos de Liderazgo de los Directivos. Formación Universitaria, 139-151.

Guisao-Paniagua, Santiago, Rincón-García, Luis David, \& Arias-Pérez, José. (2017). Capacidad de tecnologías de información y desempeño organizacional: efecto mediador de la capacidad de absorción. Cuadernos de Administración, 30(55), 3765. https://dx.doi.org/10.11144/javeriana. cao30-55.ctido

Liu, H., Ke, W., Wei, K. \& Hua, Z. (2013). The impact of IT capabilities on firm performance: The mediating roles of absorptive capacity and supply chain agility. Decision Support Systems, 54(3), 1452-1462.

https://doi.org/10.1016/j.dss.2012.12.016 
MiPyMEs, PyMES y empresas en Xalapa, Veracruz. (2017). MiPymes. Recuperado de:

www.pymes.org/municipio/Xalapa.html

Nakata, C., Zhu, Z. \& Kraimer, M. (2008). The complex contribution of information technology capability to business performance. Journal of Managerial Issues, 20(4), 485-506.

Paredes, J. (24 de Enero de 2018). opencap. Recuperado el 21 de Octubre de 2018, de opencap: http://www.opencap.mx/cuales-la-clasificacion-de-las-pymes/

Sampieri, R. H. (2006). Metodología de la investigación. México: McGraw - Hill.

Santhanam, R. \& Hartono, E. (2003). Issues in linking information technology capability to firm performance. MIS Quarterly, 27(1), 125-153. https://doi: $10.2307 / 30036521$

WEF (2015). The Global Information Technology Report 2015. Geneva. Yu, C. \& Xin-quan, G. (2011). The empirical study on the relationship between information technology capability and innovation performance: The moderating role of learning commitment. In EBusiness and E-Government (ICEE), International Conference on (pp. 1-4). IEEE 